\title{
Formation and evolution of flame kernels in autoignition of a turbulent hydrogen/air mixing layer at $50 \mathrm{~atm}$
}

\author{
Tong Yao ${ }^{1}$, Qian Wang ${ }^{2}$, Kai Hong Luo ${ }^{3 *}$ \\ ${ }^{1}$ Center for Combustion Energy, Key Laboratory for Thermal Science and Power Engineering of \\ Ministry of Education, Department of Thermal Engineering, Tsinghua University, Beijing 100084, \\ China \\ ${ }^{2}$ School of Mechanical Engineering, Shanghai Jiao Tong University, Shanghai 200240, China \\ ${ }^{3}$ Department of Mechanical Engineering, University College London, Torrington Place, London \\ WC1E 7JE, UK \\ *Corresponding Authors: qianwang@sjtu.edu.cn, k.luo@ucl.ac.uk
}

Abstract: Autoignition of a turbulent stratified mixing layer between nitrogen-diluted hydrogen and hot air under an elevated pressure of 50 atm is studied using direct numerical simulations (DNS) in this work. Homogeneous isotropic turbulence is superimposed on the flow field. Reduced chemical mechanisms and a detailed multicomponent diffusion model are employed. In addition to turbulent mixing ignition (TMI), homogeneous mixing ignition (HMI) and laminar mixing ignition (LMI) are also investigated for comparison. Autoignition chemistry over a wide range of pressures is studied in HMI and LMI, which shows different behaviors at elevated pressures versus low pressures. The importance of $\mathrm{H}_{2} \mathrm{O}_{2}$ and $\mathrm{HO}_{2}$ in TMI is highlighted as radical sinks during the ignition process and can also be used as an indicator for locating the ignition spots. Moreover, $\mathrm{OH}$ radicals can be used as a marker variable for the transition of autoignition to flame propagation under high pressure. According to the present study, turbulence has 
some influence on the radical explosion stage especially for the conservation of $\mathrm{H}_{2} \mathrm{O}_{2}$ under the elevated pressure of 50 atm. Autoignition kernels forming away from the most reactive mixture fraction isosurface are identified, which is a hybrid of autoignition and diffusive-ignition.

\section{Keywords: Direct Numerical Simulation; hydrogen; mixing layers; ignition; high pressure}

\section{Introduction}

The initiation of turbulent non-premixed combustion through autoignition is of great interest both in practical applications and fundamental combustion science. In practical combustion applications for example diesel engine, where fuel and air are injected separately, turbulent autoignition in the fuel/air mixing layer under high pressures are ubiquitous. Accurate prediction of the onset of combustion in the presence of turbulence and compositional stratifications can help to improve performance and efficiency. [1] However, turbulent autoignition in the mixing layer is difficult to model for its non-equilibrium features and intrinsic complexity involving chemical reactions, molecular and thermal diffusion, and turbulent transport. In the meantime, experimental measurements concerning the transition from unreacted state to fully burning state are very limited. Experimental data on turbulentchemistry interactions is mostly performed under atmospheric pressure and rare for high pressures. [2, 3]

On the other hand, direct numerical simulations (DNS) is a useful tool for gaining insight into autoignition in turbulent mixing layers with thermal and/or compositional stratifications [4,5] by resolving all the temporal and spatial scales. Mastorakos et al. [6] have reported results from two-dimensional (2D) DNS of methane-air mixing layers in an isotropic homogeneous decaying turbulent environment using a single-step chemistry. They observed that autoignition 
occurred at the 'most reactive' mixture fraction $\xi_{M R}$ with low magnitudes of the scalar dissipation rate $\chi$ in the fuel-air mixing layer. The role of turbulence was to create localized regions of low $\chi$ as well as high $\chi$ in the domain and, since low- $\chi$ regions favor ignition, the first occurrence of ignition in the mixing layer was increased in the presence of turbulence. This has been confirmed by extensive DNS studies at atmospheric conditions reviewed by Mastorakos in [7]. For engine relevant conditions, Mukhopadhyay and Abraham [8,9] have performed 2D DNS of autoignition of laminar and turbulent initially stratified mixing layers under 40 bar, which is representative of conditions in compression ignition engines. n-Heptane was used as the surrogate fuel and a 37-species 70-step reaction mechanism was employed in the simulations. The influence of stratification and turbulence on the two-stage ignition characteristics is investigated under elevated pressures. Bellan [10] have studied the physics of turbulent temporal mixing layer autoignition and combustion of heptane under high-pressure conditions (60-80 atm) through DNS realizations. A single-step reaction involving five species was adopted. Interactions between high-pressure and chemistry are investigated. Krisman et al [11,12] have investigated turbulent autoignition of dimethyl ether (DME)-air mixing layers by performing 2D DNS studies under $40 \mathrm{~atm}$. They found that $\xi_{M R}$ calculated from the homogeneous reactor is not sufficient to predict the location of the second stage autoignition. This is also proved in a further 3D DNS of a temporally evolving planar jet of n-heptane at 40 atm with a global chemistry [13].

Autoignition characteristics of hydrogen are markedly different from hydrocarbons. Firstly, Hydrogen has a very high diffusivity, which results in preferential diffusion, altering global combustion characteristics such as the heat release rate, turbulent flame speed and burning rate 
via thermal-diffusive instabilities [14]. In the work of Dai et al. [15], it was shown that the preferential diffusion of $\mathrm{H}_{2}$ play an important role in mixing layer ignition, and that ignition in laminar 1D mixing layer can be shorter than in homogeneous case. There are several studies focusing on autoignition of hydrogen-air mixing layers under the atmospheric pressure. Hilbert and Thevenin [16] have investigated the influence of differential diffusion and emphasized the importance of using detailed diffusion model. Im et al [17] have studied 2D DNS of hydrogenair mixtures autoigniting in an isotropic homogeneous decaying turbulence. Multi-step chemical kinetics and constant Lewis numbers were employed to model hydrogen-air oxidation. However, thermal diffusion was not considered. Hilbert and Thevenin [18] have investigated autoignition of hydrogen/air mixing layers using different diffusion models including Le number for all species, a constant Lewis number for each species and a multi-component diffusion model. They found that the onset of heat release is influenced by the diffusion. Knikkerr et al [19] compared the autoignition delay time of hydrogen-air and methane-air mixing layers using the homogeneous mixing ignition (HMI) model and a 1D linear mixing ignition (LMI) model. They concluded that detailed information is not provided by the HMI method. Considering transport effects, the LMI approach appears as a minimum requirement for the correct estimation of the ignition delay time.

Secondly, autoignition characteristics are unique for hydrogen with increasing pressures. The ignition delay curve shows a "S" shape, because different reaction pathways are dominant under various pressures [20]. However, there are few investigations on turbulent autoignition of hydrogen at elevated pressures. Most previous DNS studies of autoignition of hydrogen/air mixing layer [16-19] were performed at low pressures which lie in the regime of strong ignition. 
The role of elevated pressure on turbulent autoignition is not well demonstrated, especially with detailed chemical kinetics and multi-component transport models. At high pressures, autoignition falls into the weak ignition regime, ignition delay time becomes much longer than under the atmospheric conditions, and interactions between turbulence and chemistry are much stronger. In our previous study, we investigated autoignition of hydrogen-air mixing layers under pressures ranging from the atmospheric pressure to 30 atm [21]. In the present work, we study a time-dependent hydrogen-air igniting mixing layer using detailed models for chemistry and diffusion under an elevated pressure of 50 atm using DNS. The aim of this paper is to provide a better understanding of autoignition in thermally and compositionally stratified hydrogen/air mixtures under elevated pressures.

\section{Numerical Methods}

The turbulent autoignition process in the present study is governed by the Navier-Stokes equations, the continuity equation, and the transport equations for energy and species mass fractions. A DNS code Parcomb [22] is used to solve these equations. A spatial sixth-order central scheme and an explicit fourth-order Runge-Kutta time integrator are employed. In its recent version [23-25], the skew-symmetric formulation [26] has been implemented for the convective terms in order to reduce even further numerical dissipation and increase stability. The extended Navier-Stokes Characteristic Boundary Conditions (NSCBC) $[27,28]$ are used, with pressure relaxation applied along all open faces. The accuracy of the code has been assessed in several previous studies [16,18,21, 29-33].

The mixture fraction $\xi$ characterizes the level of mixing between the reactants. It takes a value of zero in pure oxidizer and a value of one in pure fuel. A careful definition of the mixture 
fraction $\xi$ is required when taking into account differential diffusion. The following formulation, proposed in [34] and checked for various configurations, is used in the present work.

$$
\xi=\frac{\left(Y_{H}-Y_{H, o x}\right) / 2 W_{H}-\left(Y_{O}-Y_{O, o x}\right) / W_{O}}{\left(Y_{H, f u}-Y_{H, o x}\right) / 2 W_{H}-\left(Y_{O, f u}-Y_{O, o x}\right) / W_{O}}
$$

where $Y_{H}$ and $Y_{O}$ are the elemental mass fractions of elements $\mathrm{H}$ and $\mathrm{O}$, and the subscript $o x$ and $f u$ stand for initial conditions on, respectively, the oxidizer and fuel sides. $W_{H}$ and $W_{O}$ stand for relative elemental mass for $\mathrm{H}$ and $\mathrm{O}$.

In the mixture fraction space

$$
\frac{\partial \phi}{\partial t}=\frac{\chi}{2} \frac{\partial^{2} \phi}{\partial Z^{2}}+\frac{\dot{\omega}}{\rho}
$$

$\chi$ is the instantaneous scalar dissipation rate defined as

$$
\chi=2 D(|\nabla \xi|)^{2}
$$

\section{Computational Configuration}

The DNS domain is two-dimensional with periodic and subsonic non-reflecting boundary conditions, as in [21]. The domain size is $1 \mathrm{~cm} \times 1 \mathrm{~cm}$. On the left, we impose $T=1100 \mathrm{~K}, \mathrm{Y}_{\mathrm{O} 2}=$ 0.233 and $\mathrm{Y}_{\mathrm{H} 2}=0$. On the right of the domain, we have $T=300 \mathrm{~K}, \mathrm{Y}_{\mathrm{O} 2}=0$, and $\mathrm{Y}_{\mathrm{H} 2}=0.023$. An appropriate nitrogen complement is then added, and initial velocities are set equal to zero. The initial domain and conditions are shown in Figure 1. 


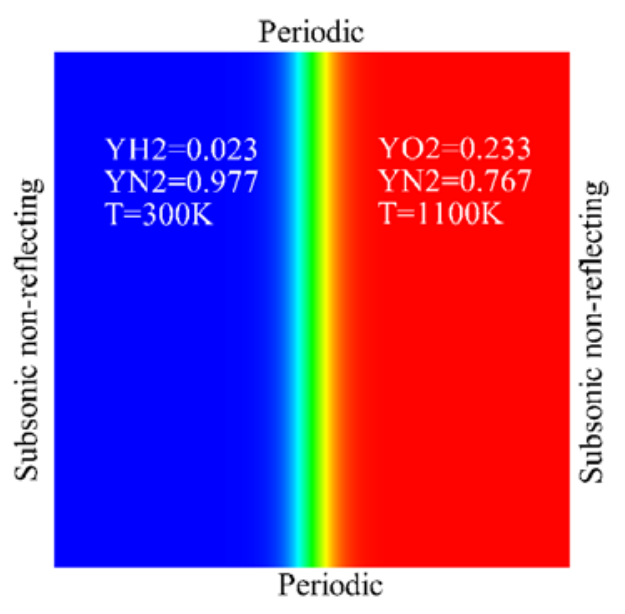

Figure 1. The computational domain and initial conditions.

The temperature and species in the mixing layer between air and fuel have hyperbolictangent profiles with a stiffness parameter. The initial values for any primitive variable e.g. mixture fraction $\xi$ are transformed into smooth profiles according to:

$$
\xi=\frac{1}{2}\left[1-\tanh \left(s \cdot\left(x-x_{m}\right)\right)\right]
$$

where $x_{m}$ is the middle of the $x$-axis. The constant $s$ is a measure of the stiffness at the fresh/burnt gas interface. The present study uses the stiffness value of $s=2000$.

A reduced chemical scheme of 9 species $\left(\mathrm{H}_{2}, \mathrm{O}_{2}, \mathrm{H}_{2} \mathrm{O}, \mathrm{OH}, \mathrm{H}, \mathrm{O}, \mathrm{HO}_{2}, \mathrm{H}_{2} \mathrm{O}_{2}\right.$, and $\left.\mathrm{N}_{2}\right)$ and 37 reactions developed by Maas et al. [35] is used to describe the combustion of $\mathrm{H}_{2}$ in the air, taking into account multicomponent diffusion velocities and thermo-diffusion effects. The multicomponent diffusion coefficients are computed from the approach proposed by Hirschfelder [36]. An approximate diffusion coefficient for the species i in the mixture is calculated. Diffusion coefficients used in the expression of the diffusion velocities in the species balance equations thus depend on the local composition of the mixture and differ for each species. This level of approximation is the one classically used in the TRANSPORT code [37] from SANDIA National Laboratories. It corresponds to a zeroth-order approximation of the complete diffusion matrix [38], 
and thus allows one to account for the difference of diffusivity between species. Note that in this so-called zeroth order approximation, a correction velocity has to be added to ensure mass conservation. Thermo-dynamic parameters are determined using fifth-order polynomial fits of experimental measurements. A field of homogeneous isotropic pseudo-turbulence is superimposed on initial profiles at $t=0$. The parameters chosen are the root-mean-square (RMS) velocity $u^{\prime}$, which indicates the turbulent intensity, and the integral length-scale of turbulence $l_{t}$.

Physical conditions investigated in the present study are listed in Table 1 . The pressure is 50 atm in this study, while pressures of 1, 5, 10 and 30 atm were considered in a previous study [21]. Turbulent intensity $u^{\prime}$ and integral length scale $l_{t}$ are set to characterize turbulence. Turbulent time scale $\tau_{t}$ and turbulent Reynolds number $R e_{t}$ are kept identical to those in [21] for different pressures. Turbulent time scale $\tau_{t}$ is $1 \mathrm{~ms}$, and the turbulent Reynolds number $R e_{t}$ is 148 . The Kolmogorov length scale $\eta$ is calculated to determine the spatial resolution of the computational domain. A grid of $5001 \times 5001$ is used, which gives a spatial resolution of $2 \mu \mathrm{m}$. The Kolmogorov scales are resolved by 3 grid points, which are adequate for convergence [39]. Time steps are determined by the CFL number.

Table 1. Turbulence conditions

\begin{tabular}{cccccccc}
\hline $\mathbf{p}(\mathbf{a t m})$ & $\mathbf{u}^{\prime}(\mathbf{m} / \mathbf{s})$ & $\mathbf{l}_{\mathbf{t}}(\mathbf{m m})$ & $\boldsymbol{\tau}_{\mathbf{t}}(\mathbf{m s})$ & $\mathbf{R e}_{\mathbf{t}}$ & $\mathbf{\eta}(\boldsymbol{\mu m})$ & Grid & Resolution $(\boldsymbol{\mu m})$ \\
\hline 50 & 0.23 & 0.23 & 1.0 & 148 & 5.4 & $5001^{2}$ & 2 \\
\hline
\end{tabular}

\section{Results and discussion}

\subsection{Homogeneous mixing ignition}

The HMI technique can be used as a simple model to investigate autoignition in the mixing 
layer without diffusion. The ignition delay time (IDT) is characterized by a period of radical buildup and the subsequent heat release with temperature increasing. IDT $t_{\text {ign }}$ can be defined by Equation (1) as the instant of inflection point of maximum heat release rate $q_{\max }$.

$$
\left.\frac{\partial^{2} q_{\max }}{\partial t^{2}}\right|_{t=t_{i g n}}=0
$$

Before investigating more complicated autoignition problems in the turbulent mixing layer, homogeneous autoignition of hydrogen/air pre-mixture is simulated first using zero-dimensional code SENKIN [40] under various pressures, temperatures and compositions. Five different Hydrogen/air combustion mechanisms [35, 41-44] are used for comparison. Figure 2 shows IDTs vary with different pressures. There are some discrepancies in the medium range of 1-10 atm. In the high-pressure regime, predictions by different mechanisms are consistent.

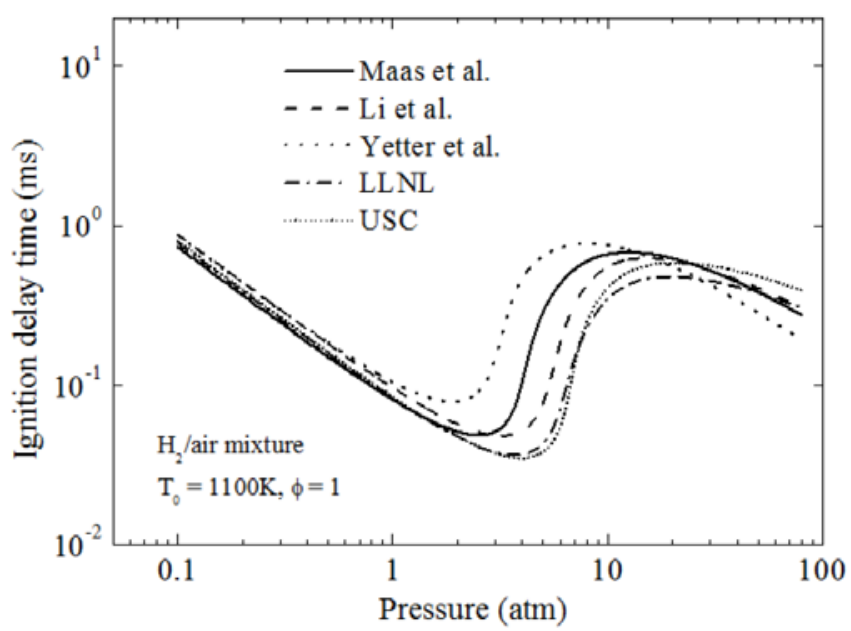

Figure 2. Calculations of autoignition time in homogeneous hydrogen-air mixtures at different pressures using various detailed mechanisms: Maas et al. [35], Li et al. [41], Yetter et al. [42], LLNL mechanism [43] and USC mechanism [44].

A batch of HMI samples is performed with different temperatures and compositions at constant pressure. Each sample of HMI can be treated as an ignition location isolated from its neighbors and left to ignite automatically. The initial profiles of mass fractions of reactants and temperature can be 
interpreted by the mixture fraction. Ignition delay time (IDT) of each sample then can be characterized by its mixture fraction $\xi$.

$$
\begin{gathered}
Y_{H}=Y_{H, f u} \xi \\
Y_{O}=Y_{O, o x}(1-\xi) \\
T=\left[c_{p, o x} T_{o x}-\xi\left(c_{p, o x} T_{o x}-c_{p, f u} T_{f u}\right)\right] /\left[c_{p, o x}(1-\xi)+c_{p, f u} \xi\right]
\end{gathered}
$$

where $Y_{H, f u}=0.023, Y_{O, o x}=0.233, T_{o x}=1100 \mathrm{~K}, T_{f u}=300 \mathrm{~K}, c_{p, o x}=Y_{O, o x} c_{p, O}+$ $\left(1-Y_{O, o x}\right) c_{p, N}, c_{p, f u}=Y_{H, f u} c_{p, H}+\left(1-Y_{H, f u}\right) c_{p, N}$.

IDTs of all samples are plotted as a function of the mixture fraction in Figure 3. It can be seen that IDT decreases from the very lean mixtures to the richer side. A minimum IDT can be found at the most-reactive mixture fraction. As the stoichiometric mixture fraction is $\xi_{\text {stoi }}=0.56$ in this case, the most reactive mixture fraction is $\xi_{M R}=0.05$ for pressure $\mathrm{p}=50 \mathrm{~atm}$. The IDT at the most reactive mixture fraction is $t_{i g n(\xi=\xi M R)}=5 \mathrm{~ms}$. Non-dimensional time is defined as $t^{*}=$ $t / t_{i g n(\xi=\xi M R)}$.

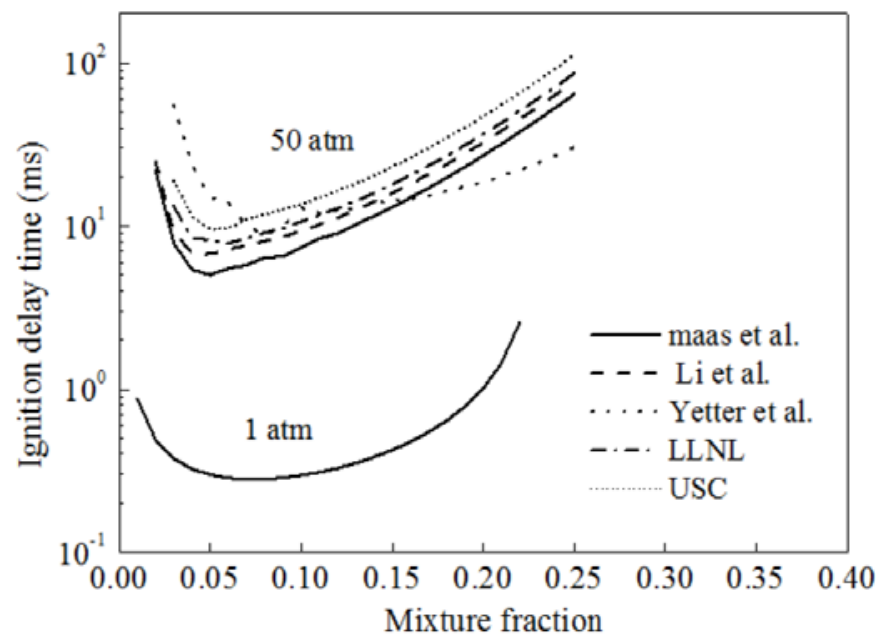

Figure 3. Autoignition delay time in HMI versus mixture fraction with various mechanisms: Maas et al. [35], Li et al. [41], Yetter et al. [42], LLNL mechanism [43] and USC mechanism [44].

Figure 4 shows the evolution of temperature and radicals under conditions corresponding to 
the most reactive mixture fraction $\xi_{M R}=0.05$. Although induction time for the accumulation of radicals decreases, thermal runway time becomes longer, which is caused by moderate heat release. It is worth noting that peak concentration of $\mathrm{HO}_{2}$ before ignition reaches the same level and corresponds to a mass fraction of $10^{-4}$. The value of $\mathrm{HO}_{2}$ peak concentration can be used as a better indicator of onset of ignition than $\mathrm{H}$ radicals at elevated pressures.

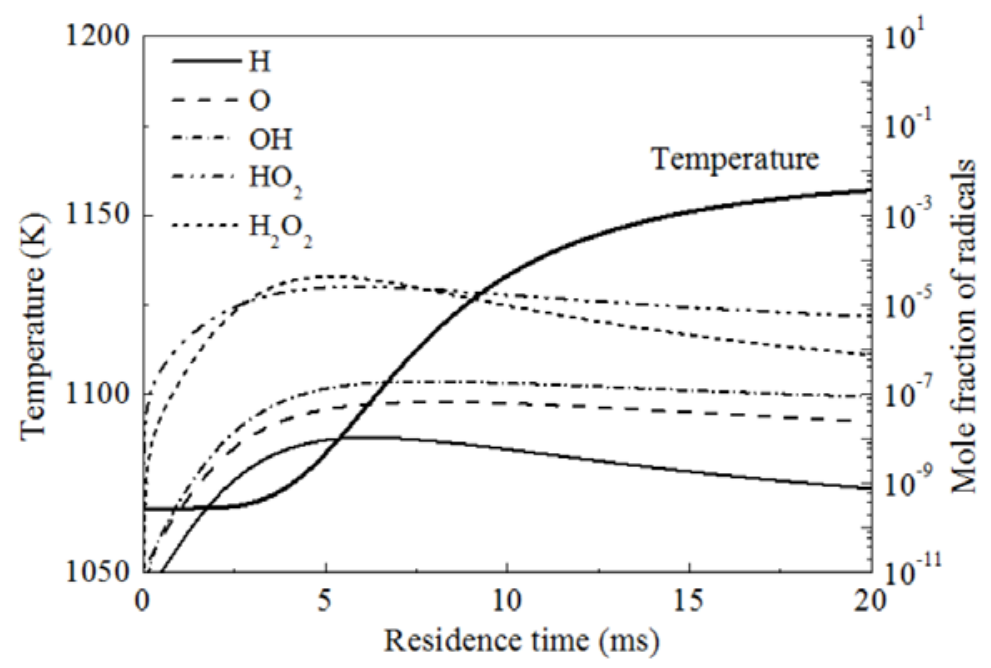

Figure 4. Evolution of temperature and radicals in HMI corresponding to the most reactive mixture fraction $\xi_{M R}=$ 0.05 .

\subsection{General description of turbulent autoignition phenomena}

Figure 5 shows the time evolution of the maximum heat release rate in turbulent and laminar cases. IDT $t_{i g n}$ of the turbulent autoignition is also defined as the instant of inflection point of maximum heat release rate $q_{\max }$ in the computational domain. This criterion indicates that ignition happens at the time instant when gradient of heat release rate reaches peak value. It can be seen from Figure 5 that turbulent autoignition is much faster that the laminar case. At the first stage of the autoignition process, the maximum heat release rate is almost the same for turbulent and laminar cases before $t^{*}=0.4$. Heat release rate for the turbulent case accelerates after $t^{*}=0.4$ and reaches its inflection point at $t^{*}=1.0$, which means a successful autoignition. However, the heat release rate 
in the laminar case grows slowly within the computation period. Turbulence accelerates the autoignition process of certain ignition kernels dramatically through decreasing the value of $\chi$, while inhibiting other kernels with increasing value of $\chi$ leading to the increased diffusive losses from the ignition location. It is worth noting that the ignition delay time of the laminar case is much longer than that of the homogeneous case. Diffusion of energy and radical species is responsible for the heat and radical losses from the ignition kernels, thus delaying the ignition.

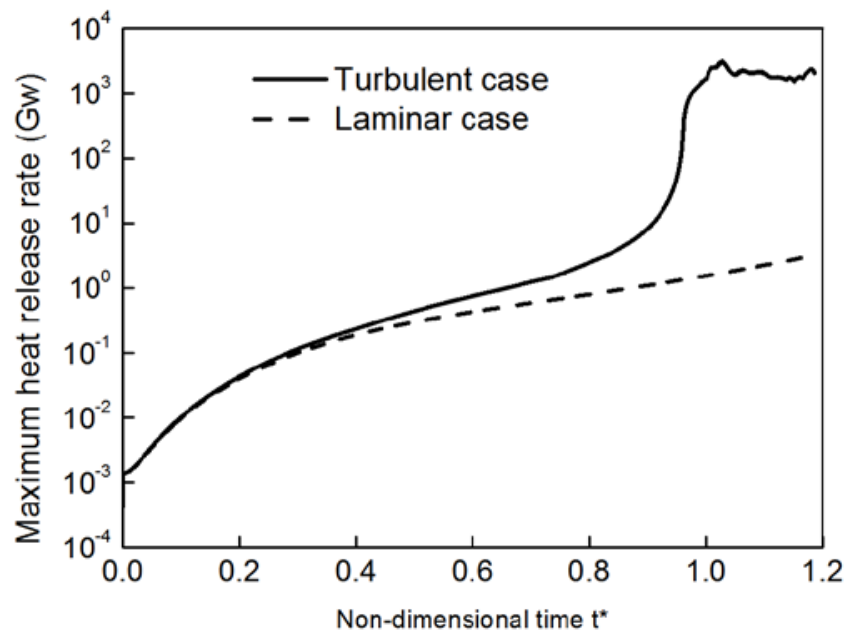

Figure 5. Comparison of evolution of maximum heat release rate for turbulent and laminar cases.

The transient evolution of the ignition process is illustrated by instantaneous images of heat release rate (HRR) and mass fractions of $\mathrm{HO}_{2}, \mathrm{H}_{2} \mathrm{O}_{2}$ and $\mathrm{OH}$ in Figures 6-9. The most reactive mixture fraction $\xi_{M R}=0.07$ iso-surface (white) and stoichiometric mixture fraction $\xi_{S T}=0.56$ iso-surface (red) are presented in the figures.

Figure 6 shows a qualitative description of HRR for a sequence of times. At $t^{*}=0.8$, we can see that ignition mainly happens in the vicinity of $\xi_{M R}=0.07$, which is richer than that of the homogeneous case. This phenomenon can be explained by the preferential diffusion of fuel, which makes ignition more likely to occur at lower temperatures (see also [21]). Four ignition kernels are 
highlighted. At $t^{*}=1.0$, we can see that Kernel $\mathrm{C}$ is the first to be successfully ignited with intense HRR and propagates to the stoichiometric $\xi_{S T}=0.56$ iso-surface. At $t^{*}=1.1$, Kernel C transforms from an ignition kernel to a triple flame. We can observe that intense HRR locates on the stoichiometric iso-surface, indicating a diffusion flame front. It is worth noting that an isolated Kernel D forms away from the most reactive mixture fraction iso-surface. Kernel $\mathrm{D}$ at $\mathrm{t}^{*}=1.1$ is characterized by a hot-spot surrounded by flame front with high heat release rate, which apparently indicates a typical autoignition kernel. However, Kernel D is not formed independently. Part of heat and radicals produced in Kernel C was transformed to the position of Kernel D at $t^{*}=1.0$ and assistant the following formation of Kernel D. This phenomenon indicates a new ignition mechanism that is different from the autoignition at the most reactive mixture fraction. Kernel D is a hybrid of autoignition and diffusive-ignition.
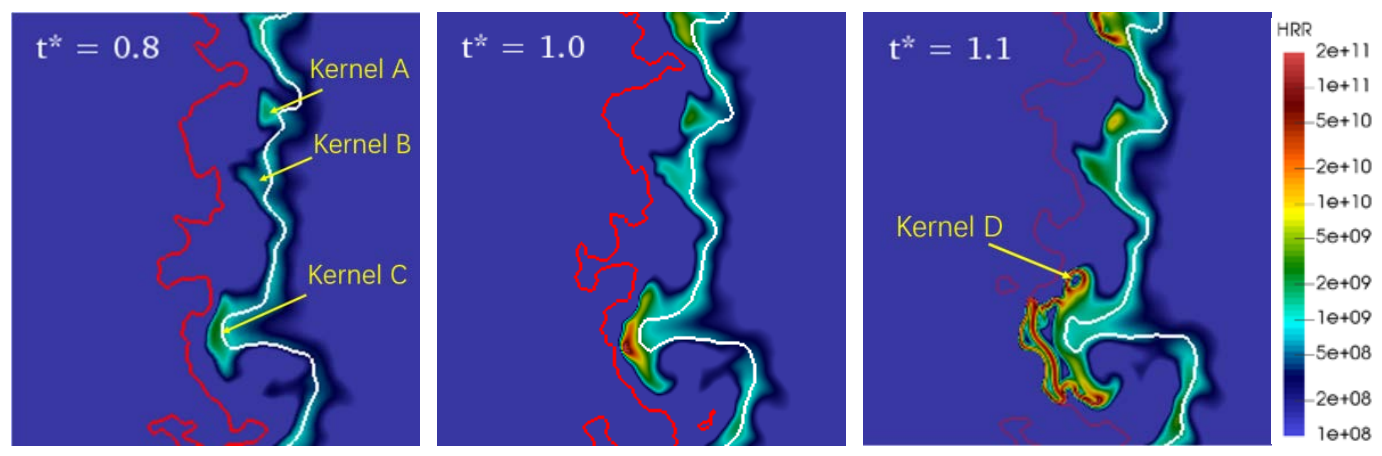

Figure 6. Instantaneous views of heat release rate (HRR) for a time sequence: $t^{*}=0.8$ (left), 1.0 (middle), and 1.1

(right). The white line represents the $\xi_{M R}=0.07$ iso-surface, while the red line represents the $\xi_{S T}=0.56$ isosurface. Three ignition kernels are highlighted.

Figure 7 shows instantaneous views of $\mathrm{HO}_{2}$ mass fraction for a sequence of times. Under high pressures $\mathrm{p}=50 \mathrm{~atm}, \mathrm{HO}_{2}$ plays an important role during the autoignition process as a sink of radicals. At $\mathrm{t}^{*}=0.8$, the distribution of $\mathrm{HO}_{2}$ is collocated with HRR in Figure 6. The HRR before ignition is mainly produced by formation of $\mathrm{HO}_{2}$. At $\mathrm{t}^{*}=1.0$, intense formation of $\mathrm{HRR}$ is observed 
at the centre of Kernel $\mathrm{C}$ where $\mathrm{HO}_{2}$ is consumed. The main contribution of energy that boosting ignition is from decomposition of $\mathrm{HO}_{2}$. At $\mathrm{t}^{*}=1.1$, the kernel develops into a lean premixed flame front and propagates to the stoichiometric iso-surface. Before autoignition $\left(t^{*}=0.8\right)$, the distribution of $\mathrm{HO}_{2}$ closely follows that of HRR, indicating that HRR prior to the autoignition is attributed to formation of $\mathrm{HO}_{2}$. At the ignition time instant $\left(\mathrm{t}^{*}=1.0\right)$, the local mass fraction of $\mathrm{HO}_{2}$ reaches a certain level, $2 \times 10^{-4}$, and then it consumes quickly to produce a peak heat release rate that indicates a successful autoignition. After ignition $\left(\mathrm{t}^{*}=1.1\right)$, the previous Kernel C develops into triple flame. We can see that $\mathrm{HO}_{2}$ is concentrated on the rich and lean premixed flame fronts. The concentration of $\mathrm{HO}_{2}$ is trivial on the diffusion flame on the stoichiometric iso-surface. Other flame kernels are at different stages of ignition. $\mathrm{HO}_{2}$ is still accumulating in Kernels A and $\mathrm{B}$. In Kernel $\mathrm{D}, \mathrm{HO}_{2}$ has been consumed and a lean premixed spherical flame has formed. The correspondence between HRR and $\mathrm{HO}_{2}$ suggests that $\mathrm{HO}_{2}$ can be used as a marker of autoignition and its mass fraction level $2 \times 10^{-4}$ is the critical value to identify the transition from autoignition to flame front propagation.
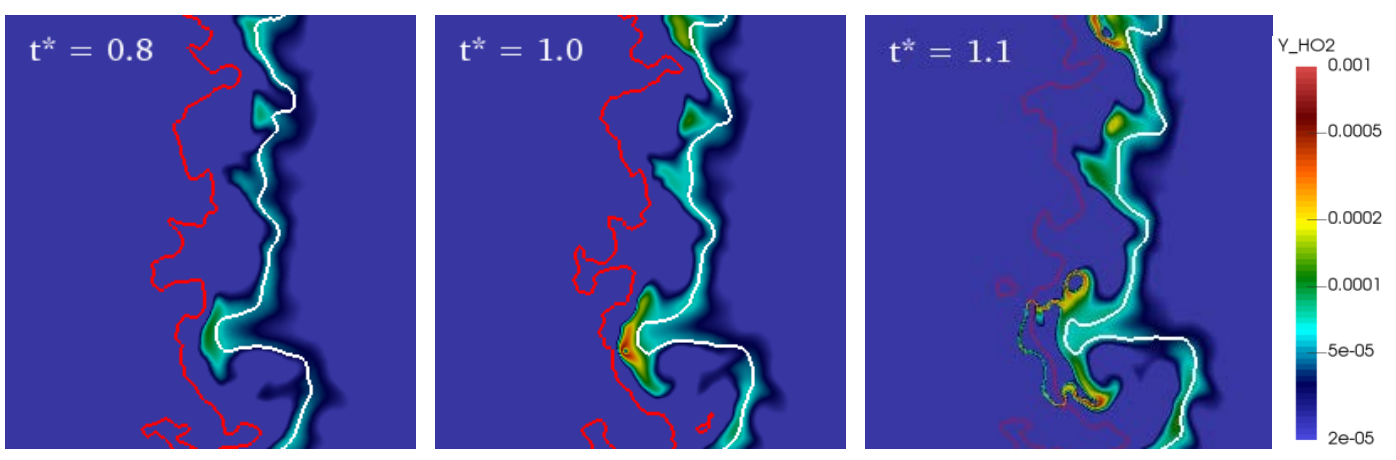

Figure 7. Instantaneous views of $\mathrm{HO}_{2}$ mass fraction (Y_HO2) for a time sequence: $\mathrm{t}^{*}=0.8$ (left), 1.0 (middle), and 1.1 (right). The white line represents the $\xi_{M R}=0.07$ iso-surface, the red line represents the $\xi_{S T}=0.56$ iso-surface.

Figure 8 shows the mass fraction distribution of $\mathrm{H}_{2} \mathrm{O}_{2}$ for different time instants. $\mathrm{H}_{2} \mathrm{O}_{2}$ also plays the role of radical sink before ignition, which is similar to $\mathrm{HO}_{2}$. We can see that $\mathrm{H}_{2} \mathrm{O}_{2}$ distributes in a wider area than that of $\mathrm{HO}_{2}$ around the ignition kernels. At $\mathrm{t}^{*}=0.8, \mathrm{H}_{2} \mathrm{O}_{2}$ 
accumulates in the richer zone away from the most reactive iso-surface $\xi_{M R}=0.07$. At $\mathrm{t}^{*}=1.0$, $\mathrm{H}_{2} \mathrm{O}_{2}$ in Kernel C has been consumed to form $\mathrm{HO}_{2}$. At $\mathrm{t}^{*}=1.1$, following localised autoignition in Kernel C and Kernel D, $\mathrm{H}_{2} \mathrm{O}_{2}$ is mainly distributed around the triple flame. In total, the concentration level of $\mathrm{H}_{2} \mathrm{O}_{2}$ is lower than that of $\mathrm{HO}_{2}$ at the ignition instant. This point will be further demonstrated later.
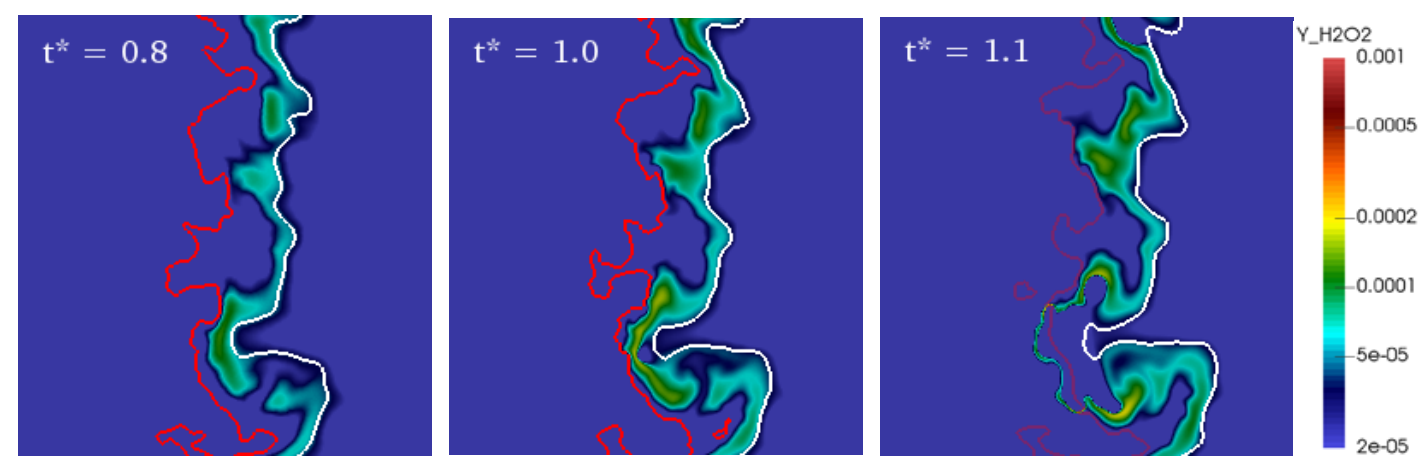

Figure 8. Instantaneous views of $\mathrm{H}_{2} \mathrm{O}_{2}$ mass fraction ( $\mathrm{Y} \_\mathrm{H} 2 \mathrm{O} 2$ ) for a time sequence: $\mathrm{t}^{*}=0.8$ (left), 1.0 (middle), and 1.1 (right). The white line represents the $\xi_{M R}=0.07$ iso-surface, while the red line represents the $\xi_{S T}=0.56$ iso-surface.

Figure 9 shows instantaneous images of $\mathrm{OH}$ for different time instants. At $t^{*}=0.8$, the $\mathrm{OH}$ concentration level is insignificant before ignition. At the ignition instant, $t^{*}=1.0$, a region of intense HRR forms in the centre of Kernel C, leading to significant formation of OH. The evolution of $\mathrm{OH}$ indicates a transition from an autoignition kernel to a propagating flame front. At $\mathrm{t}^{*}=1.1$, a continuous high temperature burning flame (triple flame) is observed with high concentration of OH locating on the stoichiometric iso-surface. In addition, the isolated ignition Kernel D evolves into a half-spherical premixed flame front with $\mathrm{OH}$ in the centre. 

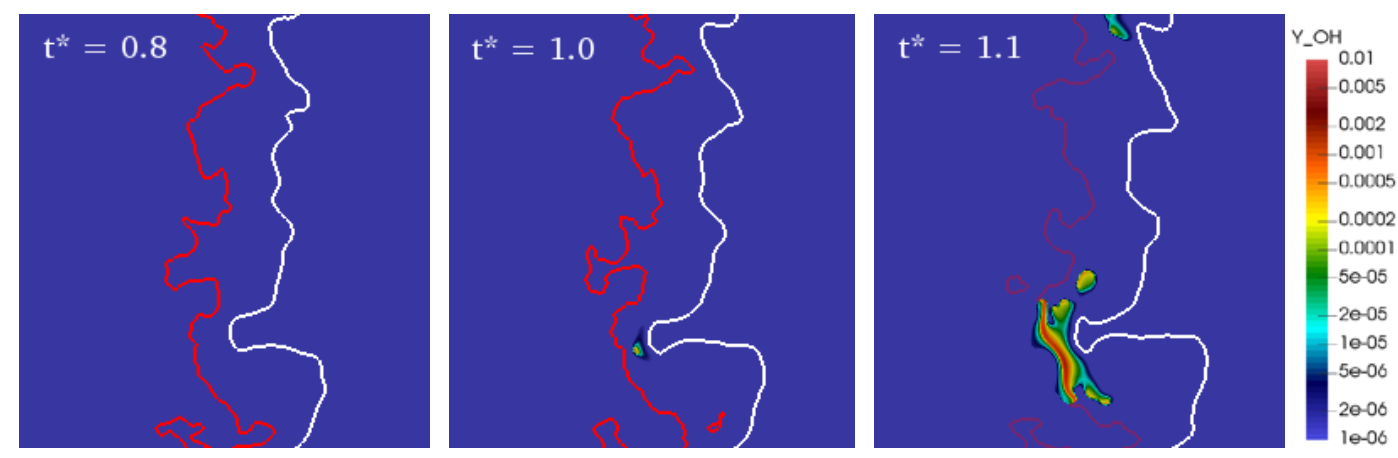

Figure 9. Instantaneous views of $\mathrm{OH}$ mass fractions for a time sequence: $\mathrm{t}^{*}=0.8$ (left), 1.0 (middle), and 1.1 (right).

The white line represents the $\xi_{M R}=0.07$ iso-surface, while the red line represents the $\xi_{S T}=0.56$ iso-surface.

\subsection{Ignition and propagation characteristics of various ignition kernels}

Evolution of the scalar dissipation rate, heat release rate and mass fractions of $\mathrm{HO}_{2}$ and $\mathrm{H}_{2} \mathrm{O}_{2}$

in different ignition kernels are presented in Figure 10. In Figure 10 (a), the scalar dissipation rate stays at a low level before ignition. Kernel C is first to ignite, followed by Kernel A. After ignition, the scalar dissipation rates in Kernel C and Kernel A begin to increase and the scalar dissipation rate in Kernel B shows no obvious rise. In Figure 10 (b), the evolution of heat release rates shows the ignition sequence in different kernels. Kernel C is the first to ignite with heat release rate increasing dramatically, followed by Kernel A and Kernel B. Figure 10 (c) shows the evolution of $\mathrm{HO}_{2}$ in different kernels. $\mathrm{HO}_{2}$ in Kernel $\mathrm{C}$ and kernel A shows similar trends. When $\mathrm{HO}_{2}$ reaches the level of $2 \times 10^{-4}$, ignition succeeds and the concentration of $\mathrm{HO}_{2}$ begins to decrease. Figure 10 (d) shows the evolution of $\mathrm{H}_{2} \mathrm{O}_{2}$ in the kernels. The concentration of $\mathrm{H}_{2} \mathrm{O}_{2}$ in kernel A and Kernel C falls quickly with the increasing of $\mathrm{HO}_{2}$, indicating a transformation from $\mathrm{H}_{2} \mathrm{O}_{2}$ to $\mathrm{HO}_{2}$. The concentration of $\mathrm{H}_{2} \mathrm{O}_{2}$ in Kernel B keeps almost the same level and decrease slowly, indicating a slow conversion of $\mathrm{H}_{2} \mathrm{O}_{2}$ to $\mathrm{HO}_{2}$, leading to a delay of ignition in Kernel B.

The scalar dissipation rate has a significant influence on turbulent autoignition. Previous studies $[6,7,21]$ have confirmed that autoignition occurs in kernels experiencing low scalar 
dissipation rates. High scalar dissipation rates can locally delay the reactions due to heat and species loss and autoignition is retarded. For the present case, low dissipation allows radicals e.g. $\mathrm{HO}_{2}$ and $\mathrm{H}_{2} \mathrm{O}_{2}$ to accumulate, and results in successful ignition. The chemical pathways of $\mathrm{H}_{2}+\mathrm{O}_{2} \rightarrow \mathrm{H}_{2} \mathrm{O}_{2}$ $\rightarrow \mathrm{HO}_{2} \rightarrow \mathrm{OH}$ in turbulent autoignition kernels can be identified. We assume that a successful transformation from $\mathrm{H}_{2} \mathrm{O}_{2}$ to $\mathrm{HO}_{2}$ is the key of ignition, when the conversion of $\mathrm{H}_{2} \mathrm{O}_{2}$ to $\mathrm{HO}_{2}$ is hindered, the ignition process is delayed, just like Kernel B shown in Figure 10.
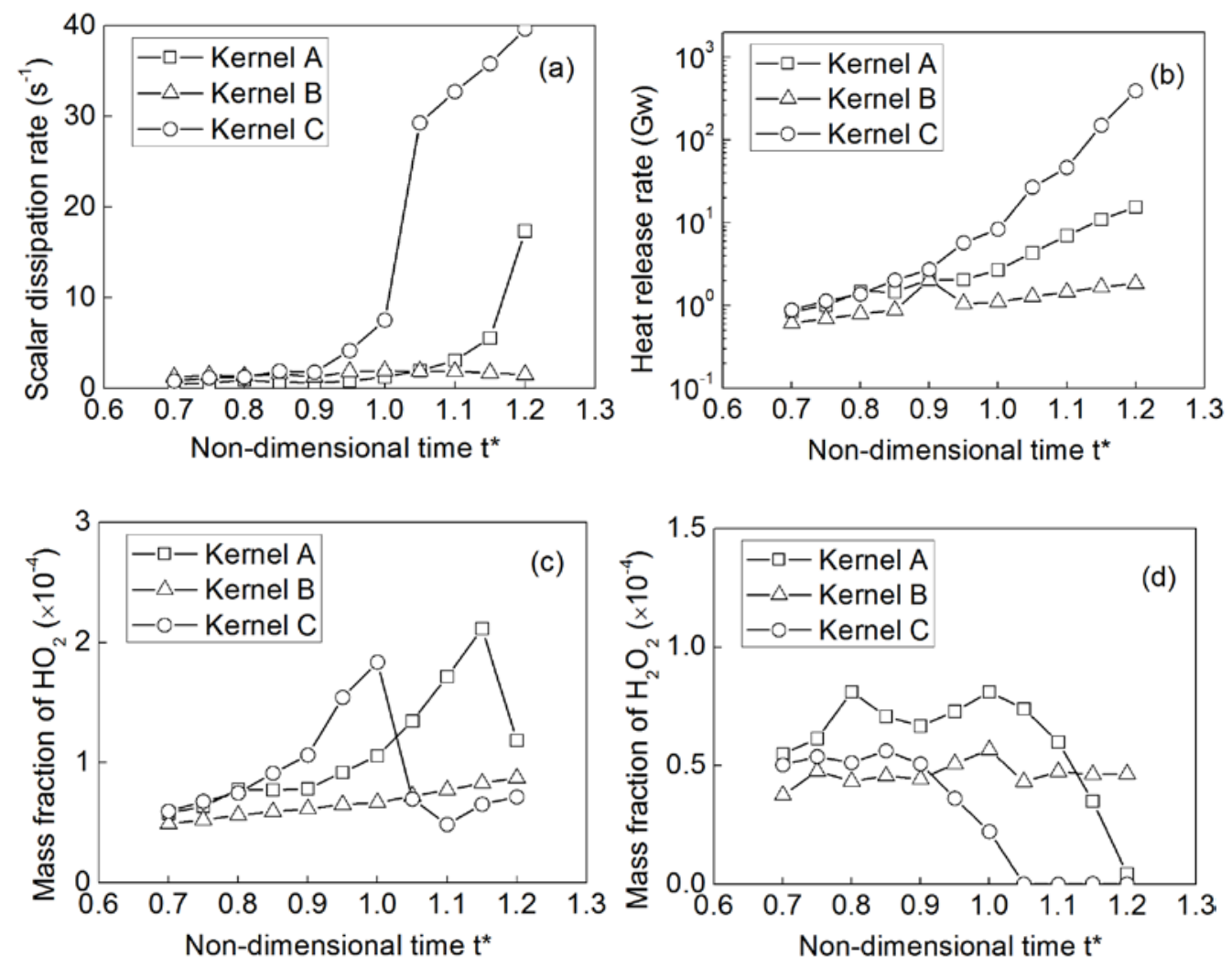

Figure 10. Evolution of different scalars in different ignition kernels: (a) scalar dissipation rate (b) heat release rate

(c) mass fraction of $\mathrm{HO}_{2}\left(\right.$ d) mass fraction of $\mathrm{H}_{2} \mathrm{O}_{2}$

\section{Conclusions}

Autoignition of a turbulent stratified mixing layer between nitrogen-diluted hydrogen and hot air under an elevated pressure of $50 \mathrm{~atm}$ has been simulated using direct numerical simulations (DNS). The present results show that distinct stages of progress exist in the autoignition of the 
hydrogen/air turbulent mixing layer at $50 \mathrm{~atm}$. 1) Before ignition, radicals build up on the most reactive mixture fraction iso-surface. $\mathrm{HO}_{2}$ and $\mathrm{H}_{2} \mathrm{O}_{2}$ accumulate with a moderate heat release rate. The distribution of $\mathrm{HO}_{2}$ is collocated with HRR. 2) At ignition, the mass fraction of $\mathrm{HO}_{2}$ in the kernels reaches the level of $2 \times 10^{-4}$, it is consumed dramatically with a high heat release rate. The kernels ignite successfully. 3)After ignition, heat release rate is distributed on a thin surface around the kernels. This stage corresponds to the formation of propagating flamelets around the autoignition kernels. Radicals like $\mathrm{HO}_{2}$ and $\mathrm{H}_{2} \mathrm{O}_{2}$ form on the front of flame. Then the lean premixed flame fronts propagate across the mixing layer to reach the stoichiometric iso-surface. Triple flames form and propagate on the stoichiometric iso-surface.

At elevated pressure of $50 \mathrm{~atm}$, autoignition chemistry in the first stage is different from that at atmospheric pressure. At low pressures, $\mathrm{H}$ radical are more important during the ignition process with the dominating chemical pathway being $\mathrm{H}_{2}+\mathrm{O}_{2} \rightarrow \mathrm{H}+\mathrm{O} \rightarrow \mathrm{OH}$, while pathways $\mathrm{H}_{2}+\mathrm{O}_{2}$ $\rightarrow \mathrm{H}_{2} \mathrm{O}_{2} \rightarrow \mathrm{HO}_{2} \rightarrow$ OH prevail at elevated pressures. Through careful examination of the evolution of the heat release rate and radicals in different Kernels, $\mathrm{HO}_{2}$ is found to be a reliable marker of ignition spots with the spike concentration of $2 \times 10^{-4}$. The delay of kernel ignition could be a slow conversion of $\mathrm{H}_{2} \mathrm{O}_{2}$ to $\mathrm{HO}_{2}$. $\mathrm{OH}$ radicals can be used as a marker variable for the transition of autoignition to flame propagation under high pressures..

Autoignition kernels (kernel D) forming away from the most reactive mixture fraction isosurface are identified for the first time. This phenomenon indicates a new ignition mechanism in auto igniting mixing layer, which is a hybrid of autoignition and diffusive-ignition.

\section{Acknowledgements}

Support from the National Natural Science Foundation of China (Grant No. 91441120) and the 
Centre for Combustion Energy at Tsinghua University is gratefully acknowledged. The simulations were performed on ARCHER funded under the EPSRC projects "UK Consortium on Mesoscale Engineering Sciences (UKCOMES)” (Grant Nos. EP/L00030X/1 and EP/R029598/1) and "High Performance Computing Support for United Kingdom Consortium on Turbulent Reacting Flow (UKCTRF)" (Grant No. EP/K024876/1). The second and third authors would like to gratefully acknowledge the support from The Royal Society - The Natural Science Foundation of China International Exchanges Scheme (Grant Nos. IE150647 and 51611130192).

\section{References}

[1] C.K. Law, Combustion physics, Cambridge: Cambridge University Press; 2006.

[2] C.N. Markides, E. Mastorakos, An experimental study of hydrogen autoignition in a turbulent coflow of heated air, Proc. Combust. Inst. 30 (2005) 883-91.

[3] Z. Wu, A.R. Masri, R.W. Bilger, An experimental investigation of the turbulence structure of a lifted H2/N2 jet flame in a vitiated co-flow, Flow Turbul. Combust. 76 (2006) 61-81.

[4] D. Veynante, L. Vervisch, Turbulent combustion modelling, Prog. Energy. Combust. Sci 28 (2002) 193-266.

[5] S. Candel, D. Thévenin, N. Darabiha, D. Veynante, Progress in numerical combustion, Combust. Sci. Tech. 149 (1999) 297-337.

[6] E. Mastorakos, T.A. Baritaud, T.J. Poinsot, Numerical simulation of autoignition in turbulent mixing flows, Combust. Flame 109 (1997) 198-223.

[7] E. Mastorakos, Ignition of turbulent non-premixed flames, Prog. Energy Combust. Sci. 35 (2009) 57-97. 
[8] S. Mukhopadhyay, J. Abraham, Influence of compositional stratification on autoignition in nheptane/air mixtures. Combust. Flame 158 (2011) 1064-1075.

[9] S. Mukhopadhyay, J. Abraham, Influence of turbulence on autoignition in stratified mixtures under compression ignition engine conditions. Proceedings of the Institution of Mechanical Engineers, Part D: Journal of Automobile Engineering 227 (2013) 748-760.

[10] J. Bellan, Direct numerical simulation of a high-pressure turbulent reacting temporal mixing layer. Combust. Flame 176 (2017) 245-262.

[11] A. Kriman, E.R. Hawkes, M. Talei, A. Bhagatwala, J.H. Chen, Polybrachial structures in dimethyl ether edge-flames at negative temperature coefficient conditions, Proc. Combust. Inst. 35 (1) (2015) 999-1006.

[12] A. Kriman, E.R. Hawkes, M. Talei, A. Bhagatwala, J.H. Chen, Characterisation of two-stage ignition in diesel engine-relevant thermochemical conditions using direct numerical simulation. Combust. Flame 172 (2016) 326-341.

[13] A. Krisman, E.R. Hawkes, J.H. Chen, J. Fluid Mech. 824 (2017) 5-41.

[14] A.N. Lipatnikov, J. Chomiak, Turbulent flame speed and thickness: phenomenology, evaluation, and application in multi-dimensional simulations, Prog. Energy Combust. Sci. 28 (2002) 1-74.

[15] P. Dai, Z. Chen, S. Chen, Ignition of methane with hydrogen and dimethyl ether addition, Fuel 118 (2014) 1-8.

[16] R. Hilbert, D. Thevenin, Influence of differential diffusion on maximum flame temperature in turbulent nonpremixed hydrogen/air flames, Combust. Flame 138 (2004) 175-187.

[17] H.G. Im, J.H. Chen, C.K. Law, Ignition of hydrogen-air mixing layer in turbulent flows, Symp. (int.) Combust. 27 (1998) 1047-1056. 
[18] R. Hilbert, D. Thévenin, Autoignition of turbulent non-premixed flames investigated using direct numerical simulations, Combust. Flame 128 (2002) 22-37.

[19] R. Knikker, A. Dauptain, B. Cuenot, T. Poinsot, Comparison of computational methodologies for ignition of diffusion layers. Combust. Sci. Tech 175 (2003) 1783-1806.

[20] D. Lee, S. Hochgreb, Hydrogen autoignition at pressures above the second explosion limit (0.6-4.0 MPa), Int. J. Chem. Kinet. 30 (1998) 385-406.

[21] T. Yao, W.H. Yang, K.H. Luo, Direct numerical simulation study of hydrogen/air auto-ignition in turbulent mixing layer at elevated pressures, Comput. Fluids (2018).

[22] D. Thévenin, F. Behrendt, U. Maas, B. Przywara, J. Warnatz, Development of a parallel direct simulation code to investigate reactive flows, Comput. Fluids 25 (1996) 485-496.

[23] D. Thévenin, G. Fru, G. Janiga, Direct numerical simulations of the impact of high turbulence intensities and volume viscosity on premixed methane flames, J Combust 2011.

[24] G. Fru, D. Thévenin, D. Markus, Direct numerical simulations of turbulent H2-Air pre-mixtures and analysis towards safety-relevant ignition prediction, Direct Large-Eddy Simul IX (2015) 525531.

[25] G. Fru, D. Thevenin, G. Janiga. Impact of turbulence intensity and equivalence ratio on the burning rate of premixed methane-air flames, Energies 4 (2011) 878-893.

[26] A.E. Honein, P. Moin, Higher entropy conservation and numerical stability of compressible turbulence simulations, J Comput Phys 201 (2004) 531-545.

[27] M. Baum, T. Poinsot, D. Thévenin, Accurate boundary conditions for multicomponent reactive flows, J Comput Phys 116 (1995) 247-261. 
[28] T. Poinsot, S. Lele, Boundary conditions for direct simulations of compressible viscous flows, J Comput Phys 101 (1992) 104-129.

[29] K.K.J. Ranga Dinesh, X. Jiang, J.A. van Oijen, Direct numerical simulation of non-premixed syngas burning with detailed chemistry, Fuel 107 (2013) 343-355.

[30] K.K.J. Ranga Dinesh, X. Jiang, J.A. van Oijen, Hydrogen-enriched non-premixed jet flames: Compositional structures with near-wall effects, International Journal of Hydrogen Energy 38 (2013) 5150-5164.

[31] K.K.J. Ranga Dinesh, X. Jiang, J.A. van Oijen, R.J.M. Bastiaans, L.P.H. de Goey, Hydrogenenriched nonpremixed jet flames: Effects of preferential diffusion, Int. J. Hydrogen Energy 38 (2013) 4848-4863.

[32] K.K.J. Ranga Dinesh, H. Shalaby, K.H. Luo, J.A. van Oijen, D. Thévenin, Effects of pressure on cellular flame structure of high hydrogen content lean premixed syngas spherical flames: A DNS study, Int. J. Hydrogen Energy 41 (2016) 21516-21531.

[33] K.K.J. Ranga Dinesh, H. Shalaby, K.H. Luo, J.A. van Oijen, D. Thévenin, Heat release rate variations in high hydrogen content premixed syngas flames at elevated pressures: Effect of equivalence ratio, Int. J. Hydrogen Energy 42 (2017) 7029-7044.

[34] R.W. Bilger, The structure of turbulent nonpremixed flames, Symp. (int.) Combust. 22 (1989) 475488.

[35] U. Maas, J. Warnatz, Ignition processes in hydrogen-oxygen mixtures, Combust. Flame 74 (1988) 53-69.

[36] J. Hirschfelder, C. Curtiss, R. Bird, Molecular Theory of Gases and Liquids, Wiley, New York, 1954. 
[37] R. Kee, G. Dixon-Lewis, J. Warnatz, M. Coltrin, J. Miller, A FORTRAN Computer Code Package for the Evaluation of Gas-Phase, Multicomponent Transport Properties, Technical Report SAND86-8246, Sandia National Laboratories, 1986.

[38] A. Ern, V. Giovangigli, Multicomponent Transport Algorithms, in: Lecture Notes in Physics, New Series M: Monographs, vol. 24, Springer-Verlag, Heidelberg, 1994.

[39] R. Yu, X.S. Bai, Direct numerical simulation of lean hydrogen/air auto-ignition in a constant volume enclosure, Combust. Flame. 160 (2013) 1706-1716.

[40] A.E. Lutz, R.J. Kee, J. A. Miller, SENKIN: A FORTRAN program for predicting homogeneous gas phase chemical kinetics with sensitivity analysis, Sandia National Laboratories Report SAND87-8248, 1988.

[41] J. Li, Z.W. Zhao, A. Kazakov, F.L. Dryer, An updated comprehensive kinetic model of hydrogen combustion, Int. J. Chem. Kinetics 36 (2004) 566-575.

[42] R.A. Yetter, H. Rabitz, R.M. Hedges, A combined stability-sensitivity analysis of weak and strong reactions of hydrogen/oxygen mixtures, Int. J. Chem. Kinetics 23 (1991) 251-278.

[43] M. O’Conaire, H.J. Curran, J.M. Simmie, W.J. Pitz, C.K. Westbrook, A comprehensive modeling study of hydrogen oxidation. International Journal of Chemical Kinetics 36 (2004) 603-622.

[44] H. Wang, X. You, A.V. Joshi, S.G. Davis, A. Laskin, F. Egolfopoulos, C.K. Law, USC Mech Version II. High-Temperature Combustion Reaction Model of H2/CO/C1-C4 Compounds, http://ignis.usc.edu/USC_Mech_II.htm, May 2007. 